\title{
Endoscopic Findings of Female Genital Tract Tuberculosis: A 3-year Analysis at a Referral Center
}

\author{
${ }^{1}$ Nutan Jain, ${ }^{2}$ Vandana Jain, ${ }^{3}$ Sonil Srivastav, ${ }^{4}$ Kriti Agarwal
}

\begin{abstract}
Objective: To study the laparoscopic and hysteroscopic appearances of female genital tract tuberculosis (FGTB).
\end{abstract}

Study design: This is a hospital-based prospective observational study.

Materials and methods: The study was conducted in the Department of Obstetrics and Gynecology, Vardhman Laparoscopy Centre, from July 1, 2014 to June 31, 2017. A total of 2,200 patients presenting with infertility were subjected to endoscopic evaluation, and the findings suggestive of TB were recorded.

Results: Out of total 2,200 patients who underwent endoscopic evaluation for infertility, 33 and $21.5 \%$ had findings suggestive of TB on laparoscopy and hysteroscopy respectively, and 59.5 and $55.6 \%$ of these patients had laparoscopic and hysteroscopic findings of early-stage disease respectively.

Conclusion: Endoscopic evaluation of unexplained infertility is an indispensable tool for early diagnosis of FGTB and providing antitubercular treatment, while it is in the reversible stage.

Keywords: Female, Genital, Hysteroscopy, Laparoscopy, Tuberculosis.

How to cite this article: Jain N, Jain V, Srivastav S, Agarwal K. Endoscopic Findings of Female Genital Tract Tuberculosis: A 3-year Analysis at a Referral Center. Int J Gynecol Endsc 2018;2(1):1-3.

Source of support: Nil

Conflict of interest: None

\section{INTRODUCTION}

Genital TB affects $12 \%$ of patients with pulmonary TB and represents 15 to $20 \%$ of extrapulmonary TB. A recent study by Indian Journal of Medical Research stated that the number of women with genital TB in infertility has increased to 30\% in 2015 from 19\% in 2011. ${ }^{1}$ It becomes symptomatic only in the end stage when the disease has caused irreparable organ damage with loss of anatomy and functional capability. Investigations like Tuberculin

\footnotetext{
${ }^{1,2}$ Consultant, ${ }^{3,4}$ Fellow

${ }^{1-4}$ Department of Gynecology and Endoscopy, Vardhman Trauma \& Laparoscopy Centre, Muzaffarnagar, Uttar Pradesh India
}

Corresponding Author: Kriti Agarwal, Fellow, Department of Gynecology and Endoscopy, Vardhman Trauma \& Laparoscopy Centre, Muzaffarnagar, Uttar Pradesh, India Phone: +919467833749, e-mail:kriti.leo@gmail.com
Skin test has sensitivity of $55 \%$ and specificity of $80 \%$ in patients with genital $\mathrm{TB},{ }^{2}$ abdominal and pelvic ultrasound, computed tomography, and magnetic resonance imaging are able to diagnose in circumstances where abdominal or pelvic mass, loculated fluid, septate ascites, thickened peritoneum, or adnexal masses are present. In such end stage, the prognosis for fertility is guarded. Hysterosalpingogram is performed frequently as an investigation for infertility. Tubal occlusion, beaded appearance, lead pipe appearance, tubal dilatation, hydrosalpinx are common findings in tubal genital TB. Polymerase chain reaction can help in early diagnosis but false negative result is an important limitation. Diagnostic laparoscopy, hysteroscopy with directed tissue, biopsy are very important and carry sensitivities up to $96 \%$. Although definitive diagnosis of genital TB requires definitive histopathological confirmation, but this can be proven in only few number of cases. Hence, Revised National Tuberculosis Control Programme (RNTCP) guidelines 2016 allow use of other lesser sensitive tests or high clinical suspicion for provisional diagnosis and starting antitubercular treatment. $^{3}$

\section{MATERIALS AND METHODS}

The study was undertaken at a tertiary care referral center for advanced gynecological laparoscopic surgery from July 1, 2014 to June 31, 2017. A total of 2,200 infertility patients underwent diagnostic laparoscopy and hysteroscopy as part of infertility evaluation. The inclusion criteria were patients complaining of infertility (primary or secondary), abnormal uterine bleeding or amenorrhea, lower abdominal pain, and adnexal mass on sonography. Past history of antitubercular treatment was noted in detail, including the duration of treatment, organs / systems involved, and the diagnostic criteria by which TB was confirmed. Previous operative interventions and operative findings were recorded in detail.

\section{RESULTS}

Tuberculosis affects a large number of people worldwide and the incidence is increasing. Genital TB is one form of extrapulmonary disease. In our study, out of total 2,200 patients who underwent diagnostic endoscopy for infertility, 33\% ( $\mathrm{n}=726)$ had findings suggestive of TB on laparoscopy and $21.5 \%(n=474)$ on hysteroscopy. The 
age of patients ranged from 20 to 40 years. The duration of infertility ranged from 1 to 25 years; $52 \%(n=378)$ and $48 \%(n=350)$ of women presented with primary and secondary infertility respectively; $8.2 \%(n=60)$ patients had history of previous ectopic pregnancy; $16.5 \%(n=120)$ patients had previous history of pulmonary TB; $59.5 \%$ $(\mathrm{n}=432)$ patients were picked up in mild form; $15.7 \%$ $(\mathrm{n}=114)$ patients in moderate; and $16.4 \%(\mathrm{n}=119)$ in severe form. In addition, $36.3 \%$ patients had milder form of TB on

Table 1: Laparoscopic findings

\begin{tabular}{lll}
\hline Observations & No. of patients & $\%$ \\
\hline $\begin{array}{l}\text { Tubes are sacculated, convoluted, fluid- } \\
\text { filled vesicles, yellow discoloration of }\end{array}$ & 432 & 59.5 \\
mesosalpinx (Fig. 1) & & \\
Hydrosalpinx (Fig. 2) & 30 & 4.1 \\
Lead pipe appearance & 12 & 1.6 \\
Encysted fluid collection & 30 & 4.1 \\
Tubo-ovarian mass & 72 & 9.9 \\
Pyosalpinx & 6 & 0.8 \\
Various grades of pelvic adhesions & 220 & 30.3 \\
Miliary tubercles (Fig. 3) & 12 & 1.6 \\
\hline
\end{tabular}

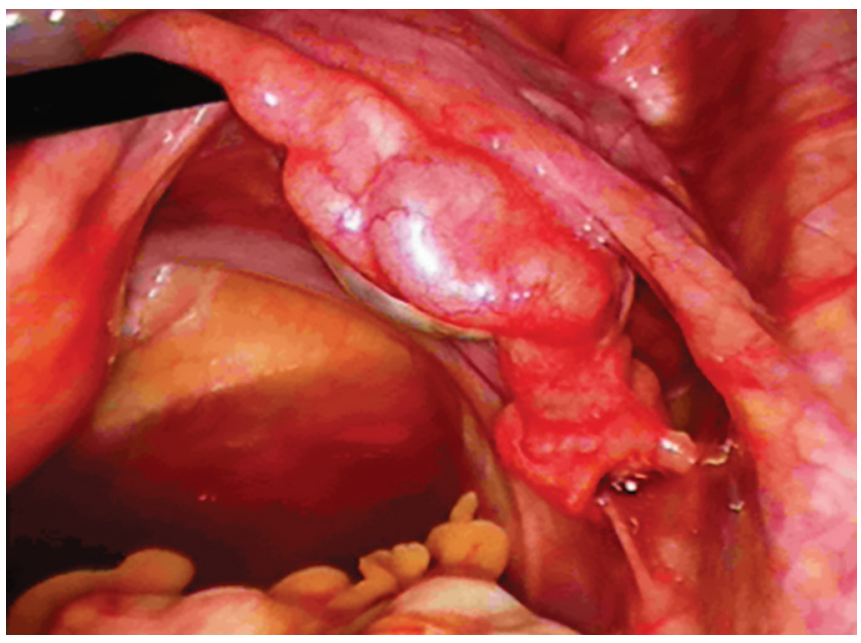

Fig. 1: Grossly convoluted and sacculated tube

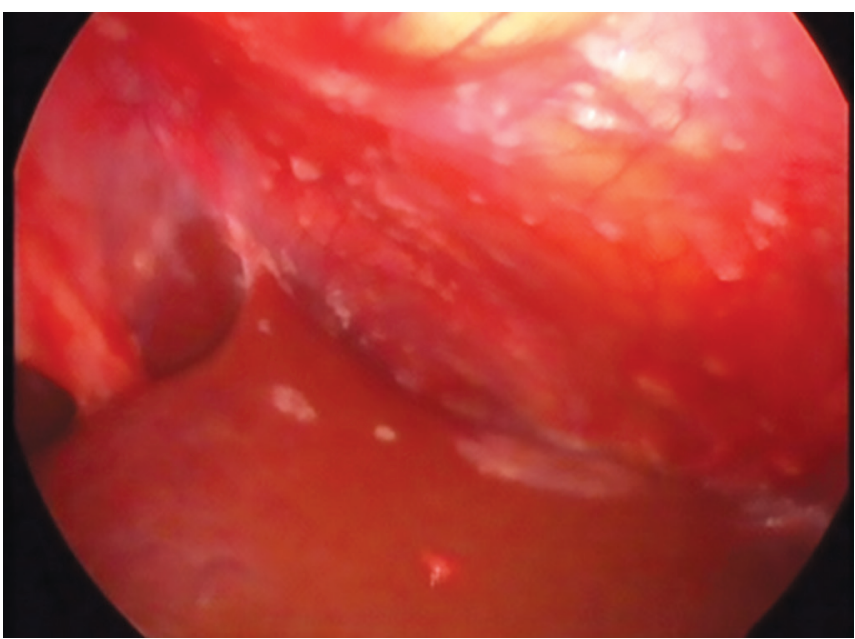

Fig. 3: Military tubercles on the liver hysteroscopy and $27.3 \%$ had moderate-to-severe form of disease. Laparoscopic and hysteroscopic findings of these patients have been tabulated in Tables 1 and 2 respectively.

\section{DISCUSSION}

Female genital TB is an important cause of significant morbidity, short- and long-term sequelae, especially infertility. Timely diagnosis and early treatment may prevent infertility and other sequelae of the disease.

Table 2: Hysteroscopic findings

\begin{tabular}{lll}
\hline Observations & No. of patients & $\%$ \\
\hline $\begin{array}{l}\text { Microcaseations and micropolyps } \\
\text { (Fig. 4) }\end{array}$ & 264 & 55.7 \\
Fibrosed ostia & 96 & \\
Synechia bands (Figs 5 and 6) & 24 & 5 \\
Narrow cavity/T-shaped cavity & 78 & 16.4 \\
Hysteroscopy not done & 12 & 2.5 \\
Asherman's syndrome & 6 & 1.2 \\
\hline
\end{tabular}

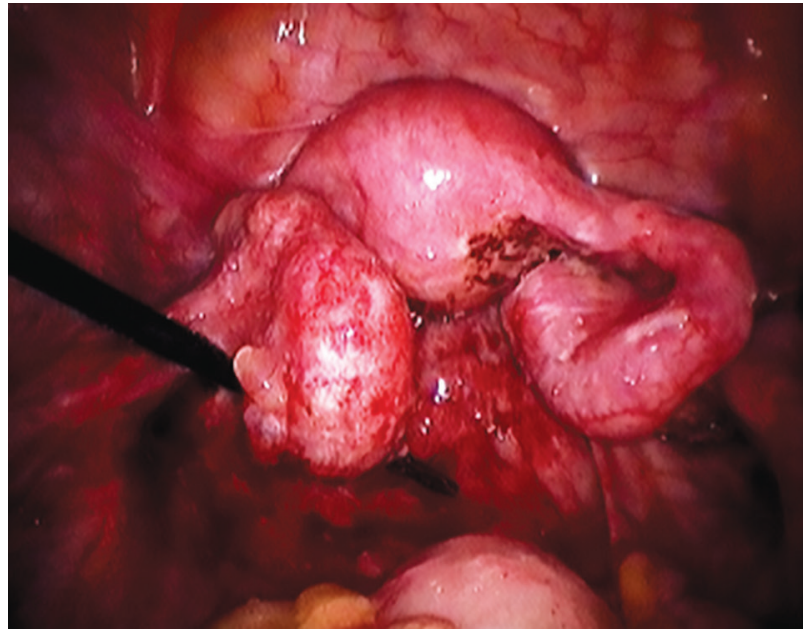

Fig. 2: Bilateral tubes tense dilated hydrosalpinx

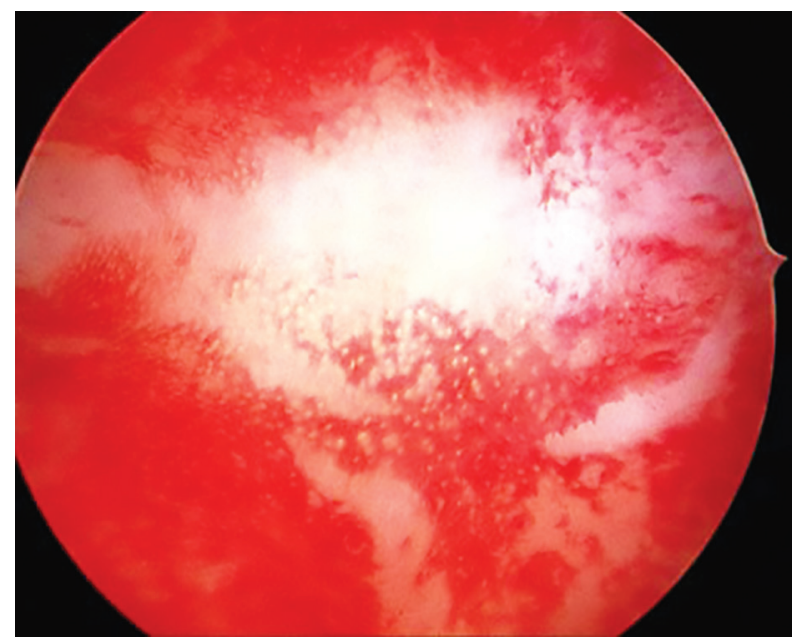

Fig. 4: Microcaseations and micropolyps 


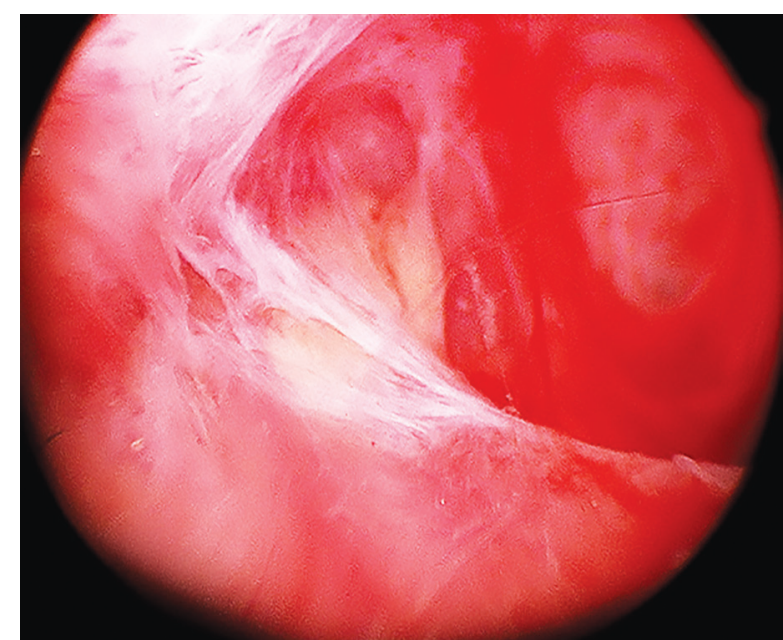

Fig. 5: Adhesion in uterine cavity

In our study, we found that 33\% (726/2200) of female factor infertility was due to genital TB, similar to $31 \%$ found in a study conducted in Yemen from 2012 to 2014 . $^{4}$ The incidence of genital TB in patients presenting with infertility was $16.4 \%$ in an Indian study of year 2005.5 Hence, the incidence of FGTB is increasing, so gynecologists will be increasingly faced with cases of TB and its consequences. In our study, 80 to $90 \%$ of cases of FGTB affects young women between 25 and 35 years of age. The median age of these patients was 31 years (22-40 years) in a study conducted in Jaipur in 2005, whereas mean age was 26.33 years in a study conducted in Surat in 2016. Hence, it is common in the reproductive age group and consequently affects the fertility potential of these women, thereby necessitating the early diagnosis and intervention of these cases to prevent the adverse reproductive sequelae of this chronic infection.

Genital TB generally occurs secondary to pulmonary (commonest) or extrapulmonary TB like gastrointestinal tract, kidneys, skeletal system, meninges, and miliary TB through hematogenous and lymphatic route. In our study, patients with previous history of pulmonary TB was $16.4 \%$ and extrapulmonary TB was $11.4 \%$, similar to 22.35 and $11.7 \%$ respectively, of a study conducted at All India Institute of Medical Sciences (AIIMS), New Delhi. ${ }^{7}$ The cases of primary infertility were $52 \%$ and secondary infertility were $47.9 \%$ in our study. The cases of primary and secondary infertility were 81.5 and $18.5 \%$ respectively, in a study in Surat in $2009 .{ }^{6}$ In our study, previous history of ectopic pregnancy was present in $8.2 \%(60 / 726)$ of patients. Genital TB was responsible for $13.2 \%$ of all cases of ectopic pregnancy. 8

Laparoscopic appearance of encysted ascites and various grades of pelvic adhesions were seen in 7.1 and $65.8 \%$ of women respectively at AIIMS, Delhi. The various findings of fallopian tubes in this study were normal looking tubes in $7.1 \%$, inability to visualize in $14.1 \%$, presence of tubercles on tubes in $3.52 \%$, caseous granuloma in

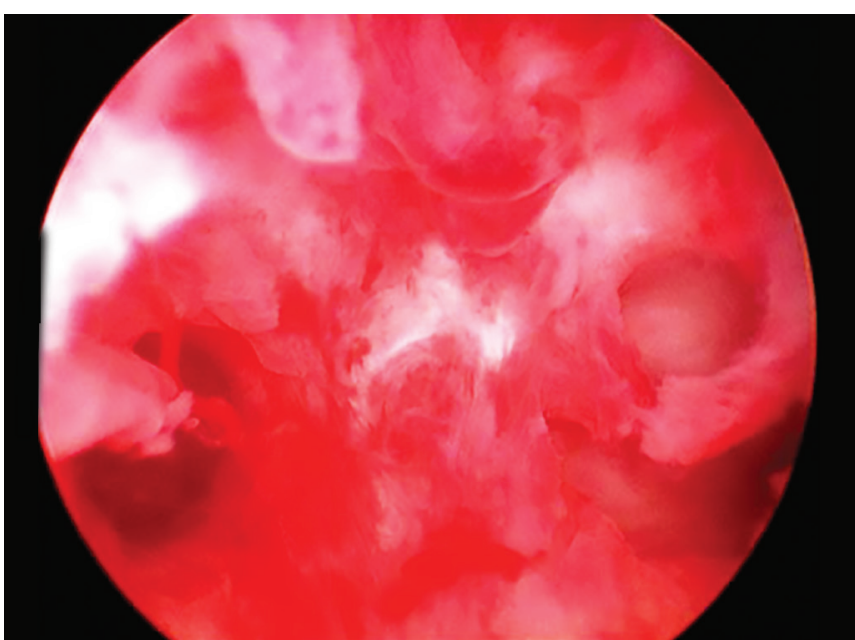

Fig. 6: Adhesion with central musculofibrous synechiae band

$3.52 \%$, hydrosalpinx in $17.6 \%$ (right tube: $11.7 \%$, left tube: $5.9 \%$ ), pyosalpinx in $3.5 \%$ on right tube, and $2.35 \%$ in left tube, beaded tube in $3.5 \%$ on right tube, $4.7 \%$ in left tube with tobacco pouch appearance in $2.35 \%$ of women. Frozen pelvis was seen in $17.2 \%$ on laparoscopy. ${ }^{7} 24.4 \%$ patients had synechiae of varying degrees on hysteroscopy. ${ }^{6}$

\section{CONCLUSION}

Female genital tract TB is still a common health problem contributing to infertility. Antitubercular therapy is not effective in restoration of women's fertility function as they often present with advanced pathology beyond recovery. Herein lies the need for early endoscopic diagnosis in order to clinch the diagnosis in reversible stage and preserve reproductive potential.

\section{REFERENCES}

1. Rajamaheswari N. Pelvic tuberculosis. Available from: www. Sunmed.org/pelvictb.html

2. Raut VS, Mahashur AA, Sheth SS. The Mantoux test in the diagnosis of genital tuberculosis in women. Int J Gynaecol Obstet 2001 Feb;72(2):165-169.

3. Technical and Operational Guidelines for TB Control in India. Chapter 3: Case finding and diagnostic strategy. New Delhi, India Central TB Division. Directorate General of Health Services Ministry of Health and Family Welfare; 2016.

4. Ali Al eryani A, Abdelrub AS, Al Harazi AH. Genital tuberculosis is common among females with tubal factor infertility: observational study. Alexandria J Med 2015 Dec;51(4):321-324.

5. Bapna N, Swarankar M, Kotia N. Genital tuberculosis and its consequences on subsequent fertility. J Obstet Gynecol India 2005 Nov-Dec;55(6):534-537.

6. Agarwal S, Chauhan J, Vachhani A, Ahir K. A study on corelation of infertility and female genital tuberculosis. Natl J Med Res 2016;6(2):151-154.

7. SharmaJB, Roy KK, PushparajM,KumarS, Malhotra N, MittalS. Laparoscopic findings in female genital tuberculosis. Arch Gynecol Obstet 2008 Oct;278(4):359-364.

8. Sharma JB, Naha M, Kumar S, Roy KK, Singh N, Arora R. Genital TB - an important cause of ectopic pregnancy in India. Indian J Tuberc 2014 Oct; 61(4):312-317. 